MITSUBISHI ELECTRIC RESEARCH LABORATORIES

http://www.merl.com

\title{
High-Dimensional Modulation for Optical Fiber Communications
}

\author{
Millar, D.S.; Koike-Akino, T. \\ TR2014-103 November 11, 2014
}

\begin{abstract}
Recent research has indicated that increasing modulation format dimensionality may provide a gain in performance over traditional 1-D, 2-D and 4-D formats. In this paper, we will discuss the principles behind high-dimensional modulation, and examine several approaches to constellation design in many dimensions.
\end{abstract}

Asia Communications and Photonics Conference (ACP) 2014

This work may not be copied or reproduced in whole or in part for any commercial purpose. Permission to copy in whole or in part without payment of fee is granted for nonprofit educational and research purposes provided that all such whole or partial copies include the following: a notice that such copying is by permission of Mitsubishi Electric Research Laboratories, Inc.; an acknowledgment of the authors and individual contributions to the work; and all applicable portions of the copyright notice. Copying, reproduction, or republishing for any other purpose shall require a license with payment of fee to Mitsubishi Electric Research Laboratories, Inc. All rights reserved. 



\title{
High-Dimensional Modulation for Optical Fiber Communications
}

\author{
David S. Millar and Toshiaki Koike-Akino \\ Mitsubishi Electric Research Laboratories, 201 Broadway, Cambridge, MA 02139, USA \\ millar@merl.com
}

\begin{abstract}
Recent research has indicated that increasing modulation format dimensionality may provide a gain in performance over traditional 1-D, 2-D and 4-D formats. In this paper, we will discuss the principles behind high-dimensional modulation, and examine several approaches to constellation design in many dimensions.
\end{abstract}

OCIS codes: (060.4080) Modulation; (060.4510) Optical communications.

\section{Introduction}

In recent years, the introduction of digital coherent detection has stimulated large amounts of research in to optimizing modulation formats in order to achieve high spectral efficiency, while maintaining good noise tolerance. A topic of significant interest over the last several years has been four-dimensional (4-D) modulation [1-5], which was initially proposed in the 1980s [6], but became a highly active research area after the topic was reintroduced by Karlsson and Agrell [7,8].

More recently, signaling in more than four dimensions has become an active research topic. While signaling in many dimensions was proposed previously in the wireless literature [9], it has recently become a topic of interest in the optical fiber communications community. Constellations have been proposed with many dimensions, utilizing wavelength [10], time-slots [11], or orthogonal optical fields [12] in order to find a basis of the required number of dimensions to represent the signal.

In this paper, we describe the concepts and subsystems underlying high-dimensional optical fiber transmission systems. Several methodologies for the design of high-dimensional signal are described, and their relative advantages and disadvantages discussed.

\section{High-Dimensional Modulation}

In our previous research [11,13-17], we have used the term "high-dimensional modulation" to refer to any modulation format with more than four dimensions. It should be noted however, that this terminology is not universal, and "multidimensional" is often used elsewhere in the literature - although this is also often used to describe 4-D modulation, which is relatively conventional in optical communications.

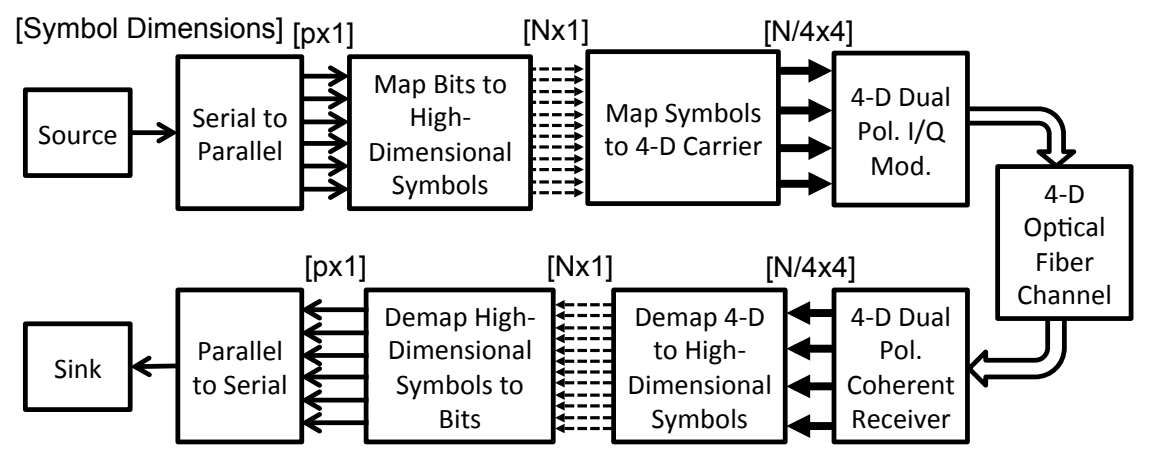

Fig. 1. Block diagram of a single carrier transmission system utilizing $N$-dimensional modulation with $p$ bits per symbol over a single-mode optical fiber channel. 
Fig. 1 shows a block diagram a transmission system utilizing high-dimensional modulation, a single optical carrier and a single mode optical channel. A number $p$ bits from a source are converted from serial to parallel, for joint modulation in $N$ dimensions. This joint modulation is essential to high-dimensional modulation, and cannot be represented as several independent modulations in lower numbers of dimensions. The $N$ dimensional signal is then mapped onto a 4-D carrier, using either multiple time-slots, multiple frequencies, or some other orthogonal basis of the required dimensionality. The optical signal is then generated, transmitted and detected in the conventional manner for digital coherent transmission systems. The $N$ component dimensions of the symbol are then demapped from the 4-D carrier, before joint demodulation of the $p$ bits in the symbol. The bits are then parallelized, before being sent to the sink.

It should be noted, that the dimensionality of the carrier does not constrain the choice of modulation dimensionality. This is due to the fact that we can use any orthogonal basis (such as time-slots, carrier frequencies etc) of appropriate dimension in order to represent the signal.

\section{Signal Design Strategies}

In this section, we describe several design strategies which have been recently used in the optical communications literature. These strategies in general, describe only the way to generate $2^{p}$ points which represent $p$ bits in $N$ dimensions. Determining an orthogonal basis for mapping $N$-D symbols to a 4-D carrier can in most cases be considered as a separate problem.

\subsection{Geometric Based Constellations}

Geometric based constellation design has previously been described in the wireless literature $[9,18]$, and more recently applied to optical communications $[13,15,17]$. The use of geometric objects such as lattices enables the relatively easy design of constellations with arbitrary numbers of points, with good performance in the high SNR regime. Unfortunately, with very high numbers of constellation points, labeling becomes a significant issue. Constellations with $2^{p}$ points have $\left(2^{p}\right)$ ! unique labelings, necessitating some manner of numerical labeling optimization in many cases.

\subsection{Block-Coded Constellations}

By using a short block code with a fixed per-dimension mapping (e.g. BPSK per dimension), it is possible to easily design constellations in many dimensions $[10,11,13,17]$. Since these constellations have a natural labeling, they do not require labeling optimization even with many constellation points. Due to the limited availability of good short block codes, the number of modulation formats which can be designed is somewhat lower when compared with geometric methods. This is particularly evident with non-binary block codes which have been used to generate constellations with higher spectral efficiency than BPSK per dimension [15].

\section{3. $\quad$ Mapping Bits Over Multiple Symbols}

Several good 4-D modulation formats are known which do not have a power of 2 number of constellation points. One design methodology which takes advantage of these formats is to encode bits over several time-slots, while forbidding certain combinations of symbols [19]. This enables the total number of allowable symbols over several time slots to be a power of two, and hence represent an integer number of bits. Binary information may therefore be easily transmitted, but symbols must be jointly detected to recover the transmitted bits. Similar to geometric constellation designs, bit labeling optimization may be challenging, and numerical optimization may be required.

\subsection{Numerical Optimization}

Constellations may also be generated using generic numerical optimization over some cost function, for example maximizing the minimum Euclidean distance between constellation points [12]. While these constellations offer more flexibility in terms of signal dimensionality than simple geometric designs, the labeling problem is not eliminated, and numerical optimization may not produce good results if the cost surface is not convex.

\subsection{Nonlinearity Optimized Constellations}

An interesting recent proposal is to optimize the constellation in order to minimize the susceptibility of the constellation to fiber nonlinearity. In [20], the $(8,4,4)$ bi-orthogonal code (as initially proposed in [10], and further examined in [17]) is used with a mapping specifically chosen to have a zero mean degree of polarization over a pair of consecutive time-slots. While the initial results from seem promising, it is not clear that this this methodology can easily be used to design other modulation formats. 


\section{Conclusions}

We have described the concepts underlying high-dimensional modulation and described the necessary subsystems for high-dimensional transmission systems in comparison to conventional systems. Several methodologies for the design of high-dimensional signal were described, and their relative advantages and disadvantages discussed.

\section{Acknowledgement}

The authors would like to thank K. Kojima, K. Parsons, T. Yoshida, T. Sugihara and T. Mizuochi for useful discussions in the development of the ideas presented in this paper.

\section{References}

1. P. Poggiolini, G. Bosco, A. Carena, V. Curri, and F. Forghieri, "Performance evaluation of coherent WDM PSQPSK (HEXA) accounting for non-linear fiber propagation effects," Opt. Express 18, 11360-11371 (2010).

2. P. Serena, A. Vannucci, and A. Bononi, "The performance of polarization switched-QPSK (PS-QPSK) in dispersion managed WDM transmissions," Proc. ECOC, Th.10.E.2, (2010).

3. D. S. Millar, D. Lavery, S. Makovejs, C. Behrens, B. C. Thomsen, P. Bayvel, and S. J. Savory, "Generation and long-haul transmission of polarization-switched QPSK at 42.9 Gb/s," Opt. Express 19, 9296-9302 (2011).

4. J. Renaudier, A. Voicila, O. Bertran-Pardo, O. Rival, M. Karlsson, G. Charlet, and S. Bigo "Comparison of SetPartitioned Two-Polarization 16QAM Formats with PDM-QPSK and PDM-8QAM for Optical Transmission Systems with Error-Correction Coding," Proc. ECOC We.1.C.5, (2010).

5. L. D. Coelho and N. Hanik, "Global Optimization of Fiber-Optic Communication Systems using FourDimensional Modulation Formats," Proc. ECOC Mo.2.B.4, (2011).

6. S. Betti, F. Curti, G. De Marchis, and E. Iannone, "Exploiting fibre optics transmission capacity: 4-quadrature multilevel signalling," Electron. Lett. 26, 992-993 (1990).

7. M. Karlsson and E. Agrell, "Which is the most power-efficient modulation format in optical links?" Opt. Express 17, 10814-10819 (2009).

8. E. Agrell and M. Karlsson, "Power-efficient modulation formats in coherent transmission systems," IEEE J. Lightwave Technology 27, 5115-5126 (2009).

9. G. D. Forney Jr, R. G. Gallager, G. Lang, F. M. Longstaff, and S. U. Qureshi, "Efficient modulation for bandlimited channels," IEEE J. Selected Areas in Communications 2, 632-647 (1984).

10. T. A. Eriksson, P. Johannisson, M. Sjödin, E. Agrell, P. A. Andrekson, and M. Karlsson, "Frequency and Polarization Switched QPSK," Proc. ECOC Th.2.D.4, (2013).

11. D. S. Millar, T. Koike-Akino, K. Kojima, and K. Parsons, "A 24-Dimensional Modulation Format Achieving 6 dB Asymptotic Power Efficiency,” Proc. SPPCOM SPM3D.6, (2013).

12. J. A. Anguita, J. Herreros, and I. B. Djordjevic, "Coherent Multimode OAM Superpositions for Multidimensional Modulation," IEEE Photonics Jour. 6, 1-11 (2014).

13. T. Koike-Akino, D. S. Millar, K. Kojima, and K. Parsons, "Eight-Dimensional Modulation for Coherent Optical Communications," Proc. ECOC Tu.3.C.3, (2013).

14. D. S. Millar, T. Koike-Akino, R. Maher, D. Lavery, M. Paskov, K. Kojima, K. Parsons, B. C. Thomsen, S. J. Savory, and P. Bayvel, "Experimental Demonstration of 24-Dimensional Extended Golay Coded Modulation with LDPC," Proc. OFC/NFOEC M3A.5, (2014).

15. D. S. Millar, T. Koike-Akino, S. Ö. Arık, K. Kojima, and K. Parsons, "Comparison of Quaternary Block-Coding and Sphere-Cutting for High-Dimensional Modulation," Proc. OFC/NFOEC M3A.4, (2014).

16. S. Ö. Arık, D. S. Millar, T. Koike-Akino,K. Kojima, and K. Parsons, "High-Dimensional Modulation for ModeDivision Multiplexing," Proc. OFC/NFOEC M3A.4, (2014).

17. D. S. Millar, T. Koike-Akino, S. Ö. Arık, K. Kojima, K. Parsons, T. Yoshida and T. Sugihara, "High-dimensional modilation for coherent optical communications systems," Opt. Express 22, 8798-8812, (2014).

18. T. Koike-Akino and V. Tarokh, "Sphere packing optimization and EXIT chart analysis for multi-dimensional QAM signaling," Proc. IEEE ICC (2009).

19. H. Bülow, "Polarization QAM modulation (POL-QAM) for coherent detection schemes," Proc. OFC/NFOEC OWG.2,(2009).

20. A. D. Shiner et al., "Demonstration of an $8 D$ Modulation Format with Reduced Inter-Channel Nonlinearities in a Polarization Multiplexed Coherent System,” arXiv:1406.1460 [physics.optics], (2014). 